EUROPHYSICS LETTERS

Europhys. Lett., (), pp. ()

\title{
The structure of colloid-polymer mixtures
}

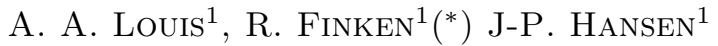 \\ 1 University Chemical Laboratories, Lensfield Rd, Cambridge CB2 1EW, UK
}

(received ; accepted )

PACS. 61.20.-p - Structure of liquids.

PACS. 82.70.Dd- Colloids.

\begin{abstract}
We investigate the structure of colloid-polymer mixtures by calculating the structure factors for the Asakura-Oosawa model in the PY approximation. We discuss the role of potential range, polymer concentration and polymer-polymer interactions on the colloid-colloid structure. Our results compare reasonably well with the recent experiments of Moussaid et. al. 55 for small wavenumber $k$, but we find that the Hansen-Verlet freezing criterion is violated when the liquid phase becomes marginal.
\end{abstract}

Suspensions of sterically stabilized colloidal particles in an organic solvent containing nonadsorbing polymer have been widely studied experimentally and theoretically because of their remarkable phase behaviour. The non-adsorbing polymer coils induce an attractive depletion interaction between the colloidal particles, the range and depth of which can be easily "tuned" by varying the polymer molecular weight (and hence its radius of gyration $R_{g}$ ) and the polymer concentration $n_{p}$. This makes polymer-colloid mixtures an important experimental model system to study the role of the range of attractive interactions on phase behaviour.

If $R_{c}$ is the radius of a spherical colloidal particle, and $\xi=R_{g} / R_{c}$ denotes the size ratio, it was predicted theoretically [1, 2], and shown experimentally [3] and by simulations [4] that the phase diagram in the $\left(\eta_{c}, n_{p}\right)$ plane (where $\eta_{c}=4 \pi n_{c} R_{c}^{3} / 3$ denotes the colloid packing fraction) exhibits colloid "gas", "liquid" and "solid" phases, when $\xi>\sim 0.3$, while the intermediate "liquid" phase becomes "marginal" for $\xi \approx 0.3$, and disappears completely for smaller size ratios $\left({ }^{1}\right)$. The previous theoretical work mostly focuses on calculating free energies and the resulting phase diagrams and no attempts were made to investigate the structure of the colloidpolymer mixtures, as embodied in the partial structure factors $S_{\alpha \beta}(k)(1 \leq \alpha \leq \beta \leq 2)$, where the indices 1 and 2 refer to the colloid and polymer species respectively). This is because the van-der-Waals like mean-field theories in[1]. 22] do not readily yield structural information, and

$\left({ }^{*}\right)$ Present address: Institut für Theoretische Physik II, Universität Düsseldorf, D-40225 Düsseldorf, Germany

$\left({ }^{1}\right)$ There is still some discrepancy between theory and simulations which predict a marginal liquid for $\xi \sim 0.4$, 4 , and experiment, which finds a marginal liquid at $\xi \sim 0.24$. 3 .

Typeset using EURO-LATEX 
because until very recently no experimental data were available. This gap has been filled by the two-colour dynamic light-scattering measurements of Moussaïd et. al. [5], which provide data for the colloid-colloid structure factor, $S(k)=S_{11}(k)$, over a limited range of wavenumbers $k$, for thermodynamic states at triple-point conditions, and for three values of the size ratio $\xi$. In particular, they concluded that under triple point conditions the amplitude of the first peak of the structure factor was always near 2.5 while the small $k$ behaviour showed a steep rise reminiscent of critical behaviour. In other words the local fluid structure at the triple point is insensitive to the potential range, while the large scale structure is sensitive to this range. These finding are analyzed here within a simple colloid-polymer model.

In this letter we apply the familiar Percus-Yevick (PY) closure [6] to the Asakura-Oosawa $(\mathrm{AO})$ [7] model of a colloid-polymer mixture to extract the partial pair distribution functions $g_{\alpha \beta}(r)$, and corresponding structure factors. The AO model treats a colloid-polymer system as a non-additive $\left({ }^{2}\right)$ mixture of spheres of radius $R_{11}=R_{c}$ (the colloids) and point particles (the weakly interacting polymer coils) which are excluded from a sphere of radius $R_{12}=$ $R_{c}+R_{g}$ around each colloid $\left(\left(^{3}\right)\right.$. This binary system is characterized by three total and direct correlation functions, $h_{\alpha \beta}(r) \equiv g_{\alpha \beta}(r)-1$, and $c_{\alpha \beta}(r)$, the Fourier transforms of which satisfy three coupled Ornstein-Zernike relations [6]:

$$
\hat{h}_{\alpha \beta}(k)=\hat{c}_{\alpha \beta}(k)+\sum_{\gamma} \rho_{\gamma} \hat{c}_{\alpha \gamma}(k) \hat{h}_{\gamma \beta}(k) .
$$

We have solved eqs (11) numerically, subject to the PY closure relations:

$$
\begin{aligned}
& g_{\alpha \beta}(r)=0, r<R_{\alpha \beta} \\
& c_{\alpha \beta}(r)=0, r>R_{\alpha \beta} .
\end{aligned}
$$

Note that (2) implies $c_{22}(r)=0$, since $R_{22}=0$ (point particles). The 2 nd and 3 rd OZ relations (11) accordingly simplify to yield the relations:

$$
\begin{aligned}
& S_{12}(k)=\sqrt{n_{1} n_{2}} \hat{h}_{12}(k)=\sqrt{n_{1} n_{2}} \hat{c}_{12}(k) S_{11}(k) \\
& S_{22}(k)=1+n_{2} \hat{h}_{22}(k)=1+n_{1} n_{2} \hat{c}_{12}^{2}(k) S_{11}(k) .
\end{aligned}
$$

Note that $S_{22}(k) \neq 1$ (and hence $h_{22}(r) \neq 0$ ), despite the fact that the polymer coils do not interact (point particles), due to correlations induced by the presence of the colloidal particles. Thus $g_{22}(r)$ provides a measure of the "void structure" of the annealed, porous "medium" created by the colloidal spheres.

Contrary to the case of additive hard-sphere mixtures [8], the coupled OZ/PY equations admit no analytic solution in the present non-additive case [9]; an analytic solution for the AO model only exists in the physically irrelevant case of negative non-additivity (which would amount to $R_{g}<0$ !) [10]. Accurate numerical solutions are, however, readily obtained and results are reported below, and compared to the experimental data and to the PY results for the effective one-component system of colloidal particles interacting via the AO depletion potential []]. The effective depletion interaction follows from the gain in free volume, accessible to the non-interacting polymer coils, due to overlaps of exclusion spheres of radius $R_{12}$ for any configuration of the non-overlapping colloid spheres of radius $R_{11}<R_{12}$. The AO pair potential follows from a second-virial-like approximation whereby only pair-wise overlaps are

$\left({ }^{2}\right)$ By non-additive we mean that the $R_{12} \neq \frac{1}{2}\left(R_{11}+R_{22}\right)$.

$\left({ }^{3}\right)$ Since interactions between polymer-coils are neglected, their activity $a_{p}=\exp \left\{\beta \mu_{p}\right\}$ is proportional to the effective polymer concentration $n_{p}^{\prime}=N_{p} / V^{\prime}$, where $V^{\prime}$ is the free volume accessible to the polymer coil; $V^{\prime}$ is a fraction $\alpha V$ of the total volume due to the presence of the colloidal particles from which the polymer coils are excluded. 
considered, thus neglecting more than two-body effective interactions resulting from overlap of three or more exclusion spheres. This is strictly valid only for $\xi<\xi_{0}=2 / \sqrt{3}-1$. For $\xi>\xi_{0}$ the two-component $\mathrm{AO}$ model can no longer be exactly mapped onto an effective one-component system of colloidal particles with strictly pairwise interactions.

For a one-component system the long wavelength $(k \rightarrow 0)$ of the structure factor $S(k)$ is given by the familiar relation[6]:

$$
\lim _{k \rightarrow 0} S(k)=n_{c} k_{B} T \chi_{T}
$$

where $\chi_{T}$ is the isothermal compressibility of the effective one-component system. The situation is more complicated in the two-component case, where the $(k \rightarrow 0)$ limit of the $\hat{c}_{\alpha \beta}(k)$ and of the $S_{\alpha \beta}(k)$ are governed by the derivatives of the chemical potentials of the two species, $\mu_{\alpha \beta}=n_{\beta}\left(\partial\left(\mu_{\alpha} / k_{B} T\right) / \partial n_{\beta}\right)_{T, n_{\alpha}}$. For the AO model in the PY approximation, where $\hat{c}_{22}(0)=1-\mu_{22} \equiv 0$, implying $\mu_{22}=1$, one finds in particular that:

$$
\lim _{k \rightarrow 0} S_{11}(k)=\frac{1}{\mu_{11}-\mu_{12} \mu_{21}},
$$

whereas (with $n=n_{1}+n_{2}$ and $x_{\alpha}=n_{\alpha} / n$ )

$$
\begin{aligned}
n k_{B} T \chi_{T} & =\lim _{k \rightarrow 0} \frac{1}{1-x_{1}^{2} n \hat{c}_{11}(k)-2 x_{1} x_{2} n \hat{c}_{12}(k)-x_{2}^{2} n \hat{c}_{22}(k)} \\
& =\frac{1}{x_{2}+x_{1}\left(\mu_{11}+2 \mu_{12}\right)},
\end{aligned}
$$

showing that the long wavelength limit of the colloid-colloid structure factor is not directly proportional to the osmotic compressibility of the colloid-polymer mixture. Note that the PY closure implies that $\mu_{22}=1$, which is tantamount to assuming that the free volume $V^{\prime}$ is unaffected by the presence of the non-interacting polymer-coils, i.e. is independent of $n_{2}$; this is precisely the basic assumption underlying the mean field theories of the phase diagram [1, 2].

The overall behaviour of the three partial structure factors is illustrated in fig. 1, where a comparison is made with the analytic PY results for an additive mixture of spheres and point-particles (corresponding to the $R_{g}=0$ limit of the AO model; $S_{11}(k)$ is then unaffected by the point particles and thus equivalent to a one-component hard-sphere model). The non-additivity is seen to strongly affect $S_{12}(k)$ and $S_{22}(k)$, while the colloid-colloid structure factor, $S_{11}(k)$, is less sensitive. The greater sensitivity of $S_{12}(k)$ and $S_{22}(k)$ may be traced back to the significant change in void structure when the excluded volume radius $R_{12}$ increases (non-additivity).

Fig. 2 2 compares $S_{11}(k)$ calculated for the AO model to the experimental data of Moussaid et.al. [5] for the three size-ratio's they used: $\xi=0.57$, which corresponds to a situation where the critical and triple points are well separated, $\xi=0.37$, where the critical point and triple point almost merge and the liquid is "marginal", and $\xi=0.24$, where there is no stable liquid region and the critical point becomes metastable w.r.t. the melting line (Note that in the experiment, $\xi=0.24$ (and not $\xi=0.37$ ) corresponds to the marginal liquid.). The colloidal packing fractions, $\eta_{c}$, and size-ratios, $\xi$, were taken from the published experimental values while the polymer packing fractions, $\eta_{p}=4 \pi n_{p} R_{g}^{3} / 3$, (which depend on $n_{p}$ and $\xi$ ) were adjusted to fit the experimental structure factors, $S_{11}(k)$, at $k=0.7 / R_{\mathrm{c}}\left(^{4}\right)$. Keeping in mind

$\left({ }^{4}\right)$ We note that taking the experimental polymer number density as fixed and varying the effective size-ratio parameter, $\xi$, would be more consistent with fitting to experiment since it is not clear that $\xi$ should be exactly equal to $R_{g} / R_{c}$. For example we find we can fit the small $k$ behaviour of $S_{11}(k)$ at the experimental polymer density for the shortest polymers by changing $\xi$ from 0.24 to 0.28 . This 

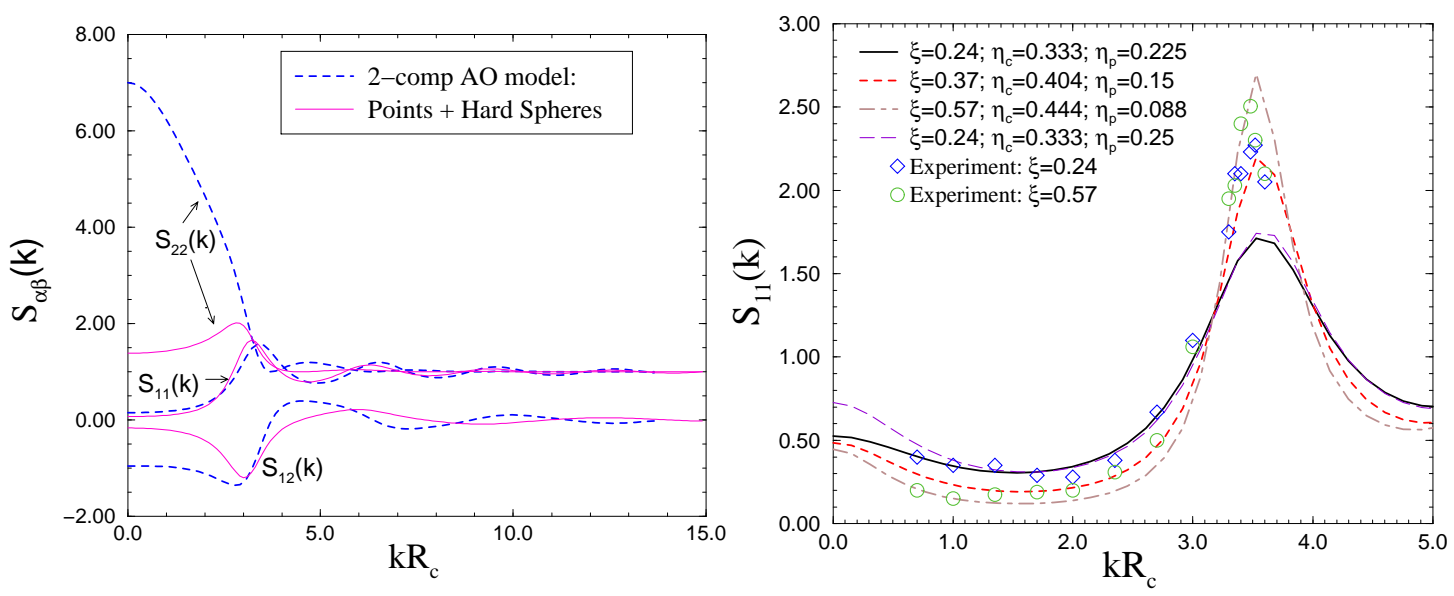

Fig. 1. - Comparison of the structure factors of the 2-component AO model and an additive mixture of hard spheres and point particles, calculated in the PY approximation for $\eta_{c}=0.333, \eta_{p}=0.1$ and $\xi=0.24$.

Fig. 2. - Comparison of the experimental colloid-colloid structure factors of Mousaïd et.al. 50 with the AO model in the PY approximation. The values of the colloid packing fraction, $\eta_{c}$, and the size ratio $\xi$ are taken from experiment. The polymer packing fraction, $\eta_{p}$, is fit to the experimental value of $S_{11}\left(k R_{c}\right)$ at the smallest accessible wavenumber $\left(k R_{c}=0.7\right)$. The resulting polymer packing fractions are $\eta_{p}=0.225,0.15$ and 0.089 respectively, compared to the measured values of $\eta_{p}=0.13,0.13$ and 0.1 respectively. The 2 nd theoretical line at $\xi=0.24$ demonstrates the insensitivity of the 1 st peak versus the sensitivity of the low $k$ behaviour of the colloid-colloid structure factor, $S_{11}(k)$, to changes in $\eta_{p}$.

the fact that PY theory tends to overestimate the amplitude of the main peak, the agreement for $\xi=0.57$ is satisfactory, but for $\xi=0.37$ and $\xi=0.24$ the amplitude of the main peak drops considerably, in contrast to the experiment where the main peak is insensitive to the size ratio $\xi$.

For a given colloidal packing fraction, $\eta_{c}$, the amplitude of the first peak of the colloid-colloid structure factor is rather insensitive to varying $\eta_{p}$ (or $\left.\xi\right)$. While shortening the range of the attractive interactions widens the melting transition, bringing the liquidus line to lower colloidal packing fractions, we find that the amplitude of the first peak of the colloid-colloid structure factor is determined primarily by the colloid packing fraction, $\eta_{c}$, and not by the proximity of the freezing transition. In contrast, the small $k$ behaviour of the structure factor is strongly affected by the proximity of a critical point or a spinodal line. These conclusions are consistent with recent simulations of one-component systems with short-range potentials 12, 13, but inconsistent with the experiments 5 .

Perhaps the most notable approximation in the AO model is the complete neglect of polymer-polymer interactions 14, 15. To obtain a rough estimate of the influence of such interactions on the pair structure of a colloid-polymer mixture we use the following model for an effective polymer-polymer pair potential:

$$
v_{22}(r)=\epsilon \exp \left(-r / 2 R_{g}\right)^{2}
$$

seems to be consistent with recent findings on the interactions between a gaussian polymer and a hard wall[11], but is inconsistent with another computer simulation study of the AO model[ [4. 

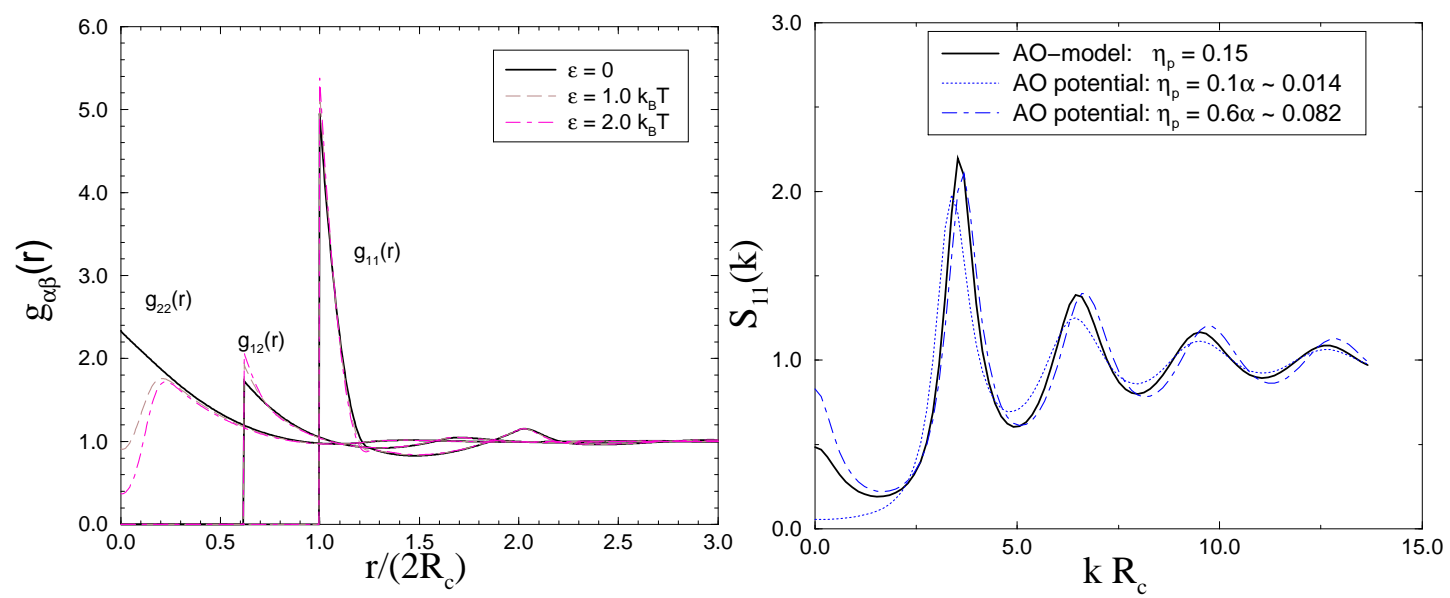

Fig. 3. - Pair distribution functions for a colloid-polymer mixture with repulsive polymer-polymer interactions of the form of equation (8) with $\eta_{c}=0.333, \eta_{p}=0.13$ and $\xi=0.24$. While the polymer-polymer radial distribution function $g_{22}(r)$ is strongly affected, the colloid-colloid radial distribution function $g_{11}(r)$ is very weakly affected by interactions.

Fig. 4. - Comparison of the colloid-colloid structure factor for the AO model and the AO potential for $\xi=0.37, \eta_{c}=0.404$ and different values of the polymer packing fraction $\eta_{p}$. The AO potential polymer fugacities are converted to polymer packing fractions through the scaled-particle free-volume factor $\alpha\left(\eta_{c}, \xi\right)$.

where $r$ is the distance between the centers of mass of the two polymer coils, and $\epsilon$ is an energy scale which is expected to be of order $k_{B} T$, since the effective interaction is largely of entropic origin. This form of the effective potential is consistent with the findings of direct simulations of hard-sphere polymer chains (16). Eq (8) is a simple parameterization of the free energy cost linked to the interpenetration of two polymer coils; the range of such an effective interaction is obviously of the order of the radius of gyration, and depending on the solvent-monomer and monomer-monomer interactions, $\epsilon$ could be positive or negative. In a good solvent $\epsilon$ is expected to be positive (effective repulsion). It is easy to incorporate the pair potential (8) in the PY theory eq. (2). The effect of turning on the polymer-polymer interactions is illustrated by the radial-distribution functions in fig. 3. The interaction (8), turns out to have a weak influence on the colloid-colloid structure, while, as expected, the polymer-polymer correlations, which are only indirect in the point particle case, are strongly affected by $v_{22}(r)$. Thus it appears that the discrepancy between the AO model and the experiments does not lie in the neglect of polymer-polymer interactions.

A final question to address is the role of many-body interactions, naturally taken into account in the two-component system, but neglected in the popular one-component approximation to the AO model: the hard-sphere system with an attractive AO potential. The comparison is somewhat complicated by thermodynamics, since the strength of the AO potential is directly proportional to the polymer activity $a_{p}$, while the AO model is most naturally interpreted in terms of the polymer density $n_{p}$ (or polymer packing fraction $\eta_{p}$ ). Since the polymers are non-interacting, however, the two can be directly related through the free-volume fraction $V^{\prime} / V=\alpha$. In fig. 4 we compare the PY results for the 2-component 
AO model in the marginal liquid state $(\xi=0.37)$ to the 1 -component $\mathrm{AO}$ potential with the $\eta_{p}$ scaled by the appropriate free-volume fraction $\alpha$ for which we take the scaled-particle approximation, which has been shown to be sufficiently accurate for the qualitative behaviour we are addressing $\left(5^{5}\right)$. Significant differences between the structure factors calculated within the one and two-component models are observed for identical values of $\eta_{p}$, particularly at small $k$. Use of the AO potential will lead to a severe underestimate of the polymer density, $n_{p}$; the discrepancy becomes progressively worse as $\xi$ increases. For example, for $\xi=0.57$ the low-k fit to the experimental $S(k)$ yields $\eta_{p}=0.088$ for the AO model but $\eta_{p} \sim 0.01$ for the AO potential. We note that the rise in the structure factor of the AO potential system is consistent with the location of the spinodal line calculated in the mean-field theories[1, 2], while the rise in the structure factor of the AO model is more consistent with the experiments. Since the same PY level of approximation is used to calculate both structure factors, the disagreement suggests the inadequacy of the AO potential or the assumption of pair-wise additivity in the reduction to an effective one-component system. It must be stressed however, that the PY closure is far from exact, and may be inadequate to cope with a short-range attractive well, as in the $\mathrm{AO}$ potential, particularly for the smaller values of $\xi$. On the other hand PY is expected to be more accurate for purely repulsive interactions as in the 2-component AO model. We are presently examining this point using thermodynamically self-consistent closures 17.

As pointed out earlier, the $k \rightarrow 0$ limits of the one-component colloid-colloid structure factor $S(k)$, and its two-component equivalent $S_{11}(k)$ do not have the same thermodynamic meaning. For $\eta_{c}=0.404, \eta_{p}=0.15$ and $\xi=0.37$, the osmotic compressibility derived from eq. (5) for the effective one-component system $\left(S(k)=S_{11}(k)\right)$, and from eq. (7) for the original two-component $\mathrm{AO}$ model are $n_{c} k_{B} T \chi_{T}=0.49$ and $n_{c} k_{B} T \chi_{T}=0.035$ respectively.

We may draw the following main conclusions from the present work:

a) In contrast to the experiments of Moussaï et.al.[5] we find (both with the 2-component AO model and the 1-component AO potential) that the first peak of the colloid-colloid structure factor and the related local fluid order is primarily determined by the colloid volume fraction, and not by the the proximity to the melting transition. Thus the Hansen-Verlet criterion, which states that the first peak of the structure factor at freezing is near 2.8 , is violated as the range of the potential is shortened to the point where the triple point vanishes. This discrepancy with experiment may be related to novel effects arising from an interplay between the triple and critical points, which for $\xi=0.24$ are in close proximity (marginal liquid).

b) In agreement with the experiments [5], we find that the large scale fluid structure and related small $k$ limit of the structure factor is strongly affected by the proximity of a spinodal line or a critical point, and thus depends significantly on the polymer density. The small $k$ limit of the structure factors is in fact well described by the AO model.

c) Polymer-polymer interactions appear to have little impact on the colloid-colloid pair structure, at least within the naive ansatz of eq. (8).

d) The one-component description, based on the effective AO pair potential, appears to be insufficient to yield a quantitatively accurate description of the colloid-colloid structure factor, particularly at small $k$. It is consistent with the phase-diagrams calculated in the mean-field theories, and similarly underestimates the polymer packing fraction for larger size ratios.

To ensure consistency of the theory, we are presently extending the PY work to other, thermodynamically consistent, integral equations which we will use to determine the phase diagram of the two-component model. We are also seeking a first principles approach to the effective polymer-polymer and polymer-colloid interactions.

$\left({ }^{5}\right)$ The overall free-volume is well represented by the scaled-particle theory expression for $\alpha \llbracket$, but the thermodynamics of theories based on free volume, such as those in ref [a], depend critically on $d^{2} \alpha / d n_{c}^{2}$, for which the scaled-particle approximation is less accurate 12 . 


\begin{abstract}
$* * *$
The authors are grateful to Wilson Poon for suggesting the PY analysis, and for providing a pre-print of ref[j, and Patrick Warren for useful suggestions. AAL would like to thank the EC for support through the grant EBRFMBICT972464.
\end{abstract}

\title{
REFERENCES
}

[1] A.P. Gast, C.K. Hall and W.B. Russel, J. Colloid Interface Sci., 96 (1983) 1437.

[2] H.N.W. Lekkerkerker, W.C.K. Poon, P.N. Pusey, A. Stroobants, and P.B. Warren, Europhys. Lett., 20 (1992) 559.

[3] S.M. Ilett, A. Orrock, W.C.K. Poon and P.N. Pusey, Phys. Rev. E, 51 (1995) 1344.

[4] E.J. MeiJer and D. Frenkel, J. Chem. Phys., 100, (1994) 6873.

[5] A. Moussaïd, W.C.K. Poon, P.N. Pusey and M.F. Soliva, Phys. Rev. Lett., 82 (1999) 225

[6] See for example J.P. Hansen and I.R. McDonald, Theory of Simple Liquids 2nd edition, (Academic Press, London) 1986.

[7] S. Asakura and F. Oosawa, J. Chem. Phys., 22 (1954) 1255;S. Asakura and F. Oosawa, J. Polym. Sci, 33 (1958) 183; A. VRIJ, Pure Appl. Chem., 48 (1976) 471.

[8] J.L. Lebowitz and J.S. Rowlinson, J. Chem. Phys., 41 (1964) 133.

[9] J.L. Lebowitz and D. Zomick, J. Chem. Phys., 54 (1971) 3335; B.N. Perry and M. Silbert, Molec. Phys., 37 (1979) 1823.

[10] D. Ronis, J. Chem. Phys., 81 (1984) 2749.

[11] E. Eisenriegler, Phys. Rev. E, 55 (1997) 3116, footnote [28].

[12] M. Dijkstra, R. van Roij, and R. Evans, Phys. Rev. E, 59 to appear (1999).

[13] G.A. Vliegenthart, J.F.M. Lodge and H.N.W. Lekkerkerker, physica A, 263 (1999) 378.

[14] P.B. Warren, S.M. Ilett, and W.C.K. Poon, Phys. Rev. E, 52 (1995) 5205.

[15] A.P. Chatterjee and K.S. Schweizer, J. Chem. Phys, 109 (1998) 10464.

[16] J. Dautenhahn and C.K. Hall, Macromolecules, 27 (1994) 5299

[17] C. Caccamo, Phys. Rep., 274 (1996) 1 